\title{
Thermodynamic entropy as a Noether invariant in a Langevin equation
}

\author{
Yuki Minami $^{1}$ and Shin-ichi Sasa ${ }^{2}$ \\ Department of Physics, Zhejiang University, Hangzhou 310027, China ${ }^{1}$, \\ Department of Physics, Kyoto University, Kyoto 606-8502, Japan ${ }^{2}$ \\ E-mail: yminami@zju.edu.cn ${ }^{1}$, sasa@scphys.kyoto-u.ac.jp ${ }^{2}$ \\ PACS numbers: 05.70.Ln,05.40.-a,
}

\begin{abstract}
We study the thermodynamic entropy as a Noether invariant in a stochastic process. Following the Onsager theory, we consider the Langevin equation for a thermodynamic variable in a thermally isolated system. By analyzing the Martin-Siggia-Rose-Janssen-de Dominicis action of the Langevin equation, we find that this action possesses a continuous symmetry in quasi-static processes, which leads to the thermodynamic entropy as the Noether invariant for the symmetry.
\end{abstract}

\section{Introduction}

Symmetry is one of the most important notions in modern physics. The symmetry reveals non-trivial laws independently of the details of systems under consideration. Well-known examples are Noether's theorem [1], the Nambu-Goldstone theorem [2, 3, 4, and the Ward-Takahashi identity [5, 6. Noether's theorem ensures the existence of a conservation law from a continuous symmetry. The Nambu-Goldstone theorem shows the appearance of gapless modes associated with a spontaneous breaking of a continuous symmetry [2, 3, 4, 7, 8, 9, 10. The Ward-Takahashi identity provides a relation among correlation functions from a symmetry.

If one assumes the converse of Noether's theorem, the existence of a conserved quantity may suggest the existence of a continuous symmetry. From this viewpoint, the thermodynamic entropy, which is a conserved quantity in quasi-static adiabatic processes, can be characterized as the Noether invariant associated with a continuous symmetry for classical Hamilton systems and quantum systems [11, 12. According to [1], in isolated classical many-body systems with an external control parameter, the action possesses a non-uniform time-translation symmetry

$$
t \rightarrow t+\eta \hbar \beta
$$

for a class of phase-space trajectories that correspond to thermodynamic processes, where $\eta$ is an infinitesimal parameter, $\hbar$ is the Planck constant, and $\beta$ is the inverse temperature that depends on a phase space point through the statistical mechanical formula. For isolated quantum many body systems with an external step protocol parameter, the effective action in a thermodynamic state space was derived under some physically reasonable assumptions [12. This action possesses a symmetry for a uniform translation of the canonical conjugate variable of the entropy. 
Here, because Langevin equations may be derived by a coarse graining method for the microscopic dynamics [13, a natural question arises: Can the entropy of a stochastic process be obtained as a Noether invariant. In this study, we find a symmetry by which the entropy is characterized as a Noether invariant of the Langevin equation that describes a stochastic process of a thermodynamic variable in thermally isolated systems. Fig. 1 1 illustrates our motivation.

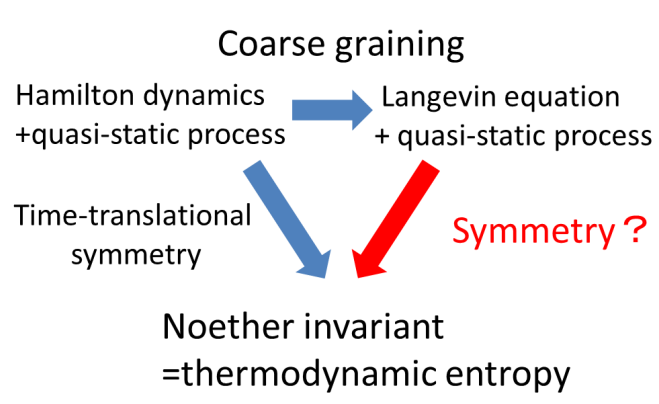

Figure 1: Illustration of the relation among symmetry, thermodynamic entropy and coarse graining.

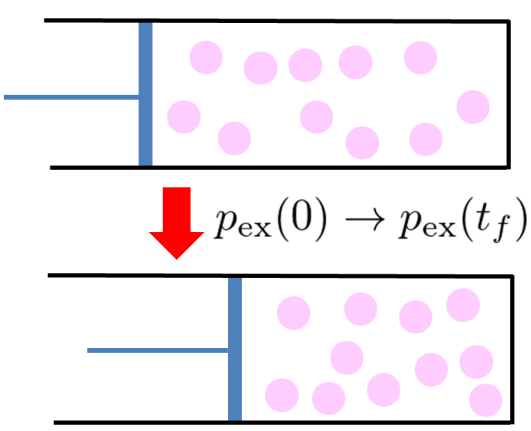

Figure 2: Particles in a container enclosed by thermally isolated walls. One side is a movable wall under pressure $p_{\text {ex }}(t)$. We externally control the pressure as a function of time: $p_{\text {ex }}(0) \rightarrow p_{\text {ex }}\left(t_{f}\right)$.

Recall that the fluctuation-dissipation theorems are understood from the time reversal symmetry [14, 15, 16, 17, 18, 19]. Similarly, the entropy conservation in quasi-static processes, which is known as the adiabatic theorem, was derived from the Hamiltonian mechanics with the reversal symmetry [20, 21, 22, 23. In this study, we prove the adiabatic theorem in a stochastic system using a fluctuation theorem similar to the Jarzynski equality [24, closely related to the time reversal symmetry. Nevertheless, the entropy cannot be described as a Noether invariant from the time reversal symmetry because it is discrete. Therefore, in this study, we explore a continuous symmetry that leads to such conservation as a result of Noether's theorem.

As a simple example, we specifically study particles in a container enclosed by thermally isolated walls. One wall is movable and externally controlled by a timedependent pressure $p_{\mathrm{ex}}(t)$, as shown in Fig. 2. According to thermodynamics, the total entropy in this system is conserved when the pressure $p_{\text {ex }}(t)$ changes in the quasi-static manner. For this system, we can describe stochastic processes of the volume by a Langevin equation. Even in this stochastic description, the total entropy is conserved in the quasi-static processes. We thus formulate a symmetry property of this stochastic system.

Technically, we derive the symmetry by employing a path integral representation, known as Martin-Siggia-Rose-Janssen-de Dominicis (MSRJD) formalism [25, 26, 27. 28, to the Langevin equation. The MSRJD formalism has the similar structure as the path integral formulation of quantum dynamics, and the theoretical arguments for the symmetry in quantum dynamics have been developed [29]. Thus, we can use techniques in the MSRJD formalism to discuss the symmetry property, as was done in [10, 30, 31]. 
The remainder of this paper is organized as follows. In Sec. 2 we explain our setup and provide the stochastic description of the system in question. Then, we derive a fluctuation theorem for our model. Using the obtained equality, we show that the entropy is conserved in the quasi-static processes. Additionally, we provide the MSRJD formalism of the Langevin equation. In Sec. 3. we first map the MSRJD formalism to Hamilton mechanics. Through the study of a Poisson bracket relation, we identify the entropy as a generator that yields a transformation. Then, we investigate whether an action in the path integral representation is symmetric under such transformation or not. In Sec. 4, we derive the Ward-Takahashi identity from the transformation generated by the entropy, and find that the symmetry breaking terms lead to a fluctuation-response relation at equal time. We also derive a thermodynamic uncertainty relation as a result of the identity. Sec. 5 concludes the paper.

Note that, throughout this paper, we set the Boltzmann constant to be unity.

\section{Setup}

\subsection{Model}

We consider $N$ particles in a container enclosed by thermally isolated walls. One side is a movable wall under pressure $p_{\text {ex }}$, as shown in Fig. 2. We first consider the case where $p_{\text {ex }}$ is constant. Let $E$ and $V$ be the internal energy of the particles and the volume of the container, while $S(E, V)$ denotes the entropy function for this system. In this case, $E$ and $V$ fluctuate while satisfying the energy conservation

$$
E+p_{\text {ex }} V=E_{\text {tot }}=\text { const, }
$$

where $E_{\text {tot }}$ corresponds to the enthalpy.

According to equilibrium thermodynamics, the probability density of $V$ is given by

$$
P_{\mathrm{eq}}\left(V ; E_{\mathrm{tot}}, p_{\mathrm{ex}}\right)=\exp \left[S\left(E_{\mathrm{tot}}-p_{\mathrm{ex}} V, V\right)-S_{*}\left(E_{\mathrm{tot}}, p_{\mathrm{ex}}\right)\right],
$$

where $S_{*}\left(E_{\mathrm{tot}}, p_{\mathrm{ex}}\right)$ is determined from the normalization of the probability. This is estimated as

$$
S_{*}\left(E_{\mathrm{tot}}, p_{\mathrm{ex}}\right)=\max _{V} S\left(E_{\mathrm{tot}}-p_{\mathrm{ex}} V, V\right)+o(N)
$$

through the saddle point calculation. $S_{*}$ is the thermodynamic entropy as a function of the enthalpy $E_{\text {tot }}$ and the pressure $p_{\text {ex }}$. Following the standard thermodynamics notation, $S_{*}\left(E_{\mathrm{tot}}, p_{\mathrm{ex}}\right)$ is also written as $S\left(E_{\mathrm{tot}}, p_{\mathrm{ex}}\right)$; however we do not use $S\left(E_{\text {tot }}, p_{\text {ex }}\right)$ to distinguish it from $S(E, V)$. From the Onsager theory, the time evolution of fluctuating $V$ is written as

$$
\frac{d V}{d t}=L \frac{d S\left(E_{\mathrm{tot}}-p_{\mathrm{ex}} V, V\right)}{d V}+\sqrt{2 L} \xi
$$

where $L$ is the Onsager coefficient and $\xi$ is the Gaussian-white noise satisfying

$$
\left\langle\xi(t) \xi\left(t^{\prime}\right)\right\rangle=\delta\left(t-t^{\prime}\right) .
$$

The stationary probability density of $V$ is equal to (3).

Now, we consider the case where $p_{\text {ex }}$ depends on time $t$. The system is then described by (5) and

$$
\frac{d E_{\mathrm{tot}}}{d t}=V \frac{d p_{\mathrm{ex}}}{d t},
$$

where the initial value $E_{\text {tot }}(0)$ is assumed. 


\subsection{Fluctuation theorem}

For the considered model, we derive a fluctuation theorem and confirm the second law

of thermodynamics. Let $\hat{V}=(V(t))_{t=0}^{t=t_{f}}$ and $\hat{p}_{\mathrm{ex}}=\left(p_{\mathrm{ex}}(t)\right)_{t=0}^{t=t_{f}}$. Then, for the fixed $V(0), E_{\text {tot }}(0)$, and $\hat{p}_{\text {ex }}$, the probability density of trajectories is given by

$$
\begin{aligned}
\mathcal{P}\left(\hat{V} \mid V(0), E_{\mathrm{tot}}(0), \hat{p}_{\mathrm{ex}}\right)= & \mathcal{N} \exp \left\{-\int_{0}^{t_{f}} d t\left[\frac{1}{4 L}\left(\frac{d V}{d t}-L \frac{d S\left(E_{\mathrm{tot}}-p_{\mathrm{ex}} V, V\right)}{d V}\right)^{2}\right.\right. \\
& \left.\left.+\frac{1}{2} \frac{d^{2} S\left(E_{\mathrm{tot}}-p_{\mathrm{ex}} V, V\right)}{d V^{2}}\right]\right\}
\end{aligned}
$$

where $\mathcal{N}$ is the normalization constant. Next, for given $\hat{V}$, $\hat{p}_{\text {ex }}$ and $E(0)$, we define the time-reversed trajectory $V^{\dagger}(t) \equiv V\left(t_{f}-t\right)$, the time reversed protocol $p_{\mathrm{ex}}^{\dagger}(t) \equiv p_{\mathrm{ex}}\left(t_{f}-t\right)$, and

$$
E^{\dagger}(0) \equiv E(0)+\int_{0}^{t_{f}} d t V(t) \frac{d p_{\mathrm{ex}}(t)}{d t}
$$

Then, we have

$$
\begin{aligned}
& \frac{\mathcal{P}\left(\hat{V}^{\dagger} \mid V^{\dagger}(0), E_{\mathrm{tot}}^{\dagger}(0), \hat{p}_{\mathrm{ex}}^{\dagger}\right)}{\mathcal{P}\left(\hat{V} \mid V(0), E_{\mathrm{tot}}(0), \hat{\mathrm{e}}_{\mathrm{ex}}\right)} \\
& =\exp \left(-\int_{0}^{t_{f}} d t \frac{d V}{d t} \frac{d S\left(E_{\mathrm{tot}}-p_{\mathrm{ex}} V, V\right)}{d V}\right) .
\end{aligned}
$$

Here,

$$
\begin{aligned}
& \frac{d S\left(E_{\mathrm{tot}}-p_{\mathrm{ex}} V, V\right)}{d t} \\
= & \frac{d V}{d t} \frac{d S\left(E_{\mathrm{tot}}-p_{\mathrm{ex}} V, V\right)}{d V}+\frac{\partial S\left(E_{\mathrm{tot}}-p_{\mathrm{ex}} V, V\right)}{\partial E_{\mathrm{tot}}}\left[\frac{d E_{\mathrm{tot}}}{d t}-\frac{d p_{\mathrm{ex}}}{d t} V\right] \\
= & \frac{d V}{d t} \frac{d S\left(E_{\mathrm{tot}}-p_{\mathrm{ex}} V, V\right)}{d V}
\end{aligned}
$$

holds according to (7). By substituting (11) into (10), we obtain

$$
\begin{aligned}
& \frac{\mathcal{P}\left(\hat{V}^{\dagger} \mid V^{\dagger}(0), E_{\mathrm{tot}}^{\dagger}(0), \hat{p}_{\mathrm{ex}}^{\dagger}\right)}{\mathcal{P}\left(\hat{V} \mid V(0), E_{\mathrm{tot}}(0), \hat{p}_{\mathrm{ex}}\right)} \\
& =\exp \left[S\left(E_{\mathrm{tot}}-V(0) p_{\mathrm{ex}}(0), V\right)-S\left(E_{\mathrm{tot}}^{\dagger}(0)-V^{\dagger}(0) p_{\mathrm{ex}}^{\dagger}(0), V^{\dagger}(0)\right)\right] \cdot(12
\end{aligned}
$$

This can be rewritten using (3) as

$$
\begin{aligned}
& \frac{\mathcal{P}\left(\hat{V}^{\dagger} \mid V^{\dagger}(0), E_{\mathrm{tot}}^{\dagger}(0), \hat{p}_{\mathrm{ex}}^{\dagger}\right) P_{\mathrm{eq}}\left(V^{\dagger}(0) ; E_{\mathrm{tot}}^{\dagger}(0), p_{\mathrm{ex}}^{\dagger}(0)\right)}{\mathcal{P}\left(\hat{V} \mid V(0), E_{\mathrm{tot}}(0), \hat{p}_{\mathrm{ex}}\right) P_{\mathrm{eq}}\left(V(0) ; E_{\mathrm{tot}}(0), p_{\mathrm{ex}}(0)\right)} \\
& =\exp \left[-\left(S_{*}\left(E_{\mathrm{tot}}(0), p_{\mathrm{ex}}(0)\right)-S_{*}\left(E_{\mathrm{tot}}(0), p_{\mathrm{ex}}(0)\right)\right)\right],
\end{aligned}
$$

Because the expectation of $A(\hat{V})$ for any function of $\hat{V}$ is written as

$$
\langle A\rangle \equiv \int \mathcal{D} \hat{V} \mathcal{P}\left(\hat{V} \mid V(0), E_{\mathrm{tot}}(0), \hat{p}_{\mathrm{ex}}\right) P_{\mathrm{eq}}\left(V(0) ; E_{\mathrm{tot}}(0), p_{\mathrm{ex}}(0)\right) A(\hat{V}),
$$

we obtain

$$
\left\langle\exp \left[-\left(S_{*}\left(E_{\mathrm{tot}}\left(t_{f}\right), p_{\mathrm{ex}}\left(t_{f}\right)\right)-S_{*}\left(E_{\mathrm{tot}}(0), p_{\mathrm{ex}}(0)\right)\right)\right]\right\rangle=1 .
$$


This is in the same form as the integral fluctuation theorem [32, while its mathematical structure is basically equivalent to the Jarzynski equality 24 .

Because $S_{*}\left(E_{\mathrm{tot}}(0), p_{\mathrm{ex}}(0)\right)$ is independent of $\hat{V}$, we also have

$$
\left\langle\exp \left[-S_{*}\left(E_{\mathrm{tot}}\left(t_{f}\right), p_{\mathrm{ex}}\left(t_{f}\right)\right)\right]\right\rangle=\exp \left[-S_{*}\left(E_{\mathrm{tot}}(0), p_{\mathrm{ex}}(0)\right)\right] .
$$

From $e^{-x} \geq 1-x$ for any real number $x$, we find

$$
\left\langle S_{*}\left(E_{\mathrm{tot}}\left(t_{f}\right), p_{\mathrm{ex}}\left(t_{f}\right)\right)\right\rangle \geq S_{*}\left(E_{\mathrm{tot}}(0), p_{\mathrm{ex}}(0)\right),
$$

which is the second law of thermodynamics.

\subsection{Quasi-static processes}

When the time scale of change in $p_{\text {ex }}(t)$ is much longer than that of the relaxation time of thermodynamic quantities in the system, such an operation is called quasistatic operation. Explicitly, we assume that the relaxation time is one by appropriate dimensionless forms, and we introduce a small parameter $\epsilon$ by which we can express the protocol $p_{\text {ex }}(t), 0 \leq t \leq t_{f}$, as

$$
\begin{aligned}
& p_{\mathrm{ex}}(t)=\bar{p}_{\mathrm{ex}}(\epsilon t), \\
& t_{f}=\frac{\tau_{f}}{\epsilon},
\end{aligned}
$$

where the functional form of $\bar{p}_{\mathrm{ex}}$ and $\tau_{f}$ are independent of $\epsilon$.

Next, we take $\Delta$ such that $1 \ll \Delta \ll \epsilon^{-1}$ and define

$$
\bar{V}_{n} \equiv \frac{1}{\Delta} \int_{n \Delta}^{(n+1) \Delta} d t V(t),
$$

to express

$$
\begin{aligned}
E_{\mathrm{tot}}\left(t_{f}\right)-E_{\mathrm{tot}}(0) & =\int_{0}^{t_{f}} d t V(t) \frac{d}{d t} \bar{p}_{\mathrm{ex}}(\epsilon t), \\
& =\sum_{n} \epsilon \Delta \bar{V}_{n} \bar{p}_{\mathrm{ex}}^{\prime}\left(\tau_{n}\right)+O\left((\epsilon \Delta)^{2}\right), \\
& =\int_{0}^{\tau_{f}} d \tau \bar{V}(\tau) \bar{p}_{\mathrm{ex}}^{\prime}(\tau),
\end{aligned}
$$

where $\tau_{n}=n \Delta \epsilon$ and $\bar{V}\left(\tau_{n}\right) \equiv \bar{V}_{n}$. By applying the law of large numbers to (20), we conclude that the fluctuations of $\bar{V}(\tau)$ tend to zero in the limit $\Delta \gg 1$, and, consequently, $E_{\text {tot }}\left(t_{f}\right)-E_{\text {tot }}(0)$ takes a typical value with high probability in the quasi-static limit. Therefore, (16) leads to the entropy conservation:

$$
S_{*}\left(E_{\mathrm{tot}}\left(t_{f}\right), p_{\mathrm{ex}}\left(t_{f}\right)\right)=S_{*}\left(E_{\mathrm{tot}}(0), p_{\mathrm{ex}}(0)\right) .
$$

\subsection{MSRJD formalism}

By introducing an auxiliary variable $\pi$, we can express (8) as

$$
\begin{aligned}
\mathcal{P}\left(\hat{V} \mid V(0), E_{\mathrm{tot}}(0), \hat{p}_{\mathrm{ex}}\right) & \\
=\mathcal{N}^{\prime} \int \mathcal{D} & \pi \exp \left\{-\int_{0}^{t_{f}} d t L\left[\pi+\frac{i}{2 L}\left(\frac{d V}{d t}-L \frac{d S\left(E_{\mathrm{tot}}-p_{\mathrm{ex}} V, V\right)}{d V}\right)\right]^{2}\right\} \\
& \exp \left\{-\int_{0}^{t_{f}} d t\left[\frac{1}{4 L}\left(\frac{d V}{d t}-L \frac{d S\left(E_{\mathrm{tot}}-p_{\mathrm{ex}} V, V\right)}{d V}\right)^{2}\right.\right. \\
+ & \left.\left.\frac{1}{2} \frac{d^{2} S\left(E_{\mathrm{tot}}-p_{\mathrm{ex}} V, V\right)}{d V^{2}}\right]\right\},
\end{aligned}
$$


which is further rewritten as

$$
\mathcal{P}\left(\hat{V} \mid V(0), E_{\text {tot }}(0), \hat{p}_{\mathrm{ex}}\right)=\mathcal{N}^{\prime} \int \mathcal{D} \pi \exp \left[I_{\mathrm{MSR}}\right]
$$

where

$$
\begin{aligned}
I_{\mathrm{MSR}}[V, \pi]= & \int_{0}^{t_{f}} d t\left[-i \pi\left(\frac{d V}{d t}-L \frac{d S\left(E_{\mathrm{tot}}-p_{\mathrm{ex}} V, V\right)}{d V}\right)-L \pi^{2}\right. \\
& \left.-\frac{1}{2} \frac{d^{2} S\left(E_{\mathrm{tot}}-p_{\mathrm{ex}} V, V\right)}{d V^{2}}\right] .
\end{aligned}
$$

For any observable $\mathcal{O}$ that is a function of $\hat{V}$, the expectation of $\mathcal{O}$ is expressed as

$$
\langle\mathcal{O}\rangle \equiv \int \mathcal{D} \pi \mathcal{D} V \exp \left[I_{\mathrm{MSR}}\right] \mathcal{O},
$$

where the normalization constant is absorbed into the integral measure to satisfy $\langle 1\rangle=1$. The path integral representation (26) is called the MSRJD formalism.

In order to see a physical interpretation of the auxiliary variable $\pi$, we add a perturbation $f_{p}(t)$ to the right-hand side of (5) and consider the expectation of a quantity $A\left(t^{\prime}\right)$ at time $t^{\prime}$ denoted by $\left\langle A\left(t^{\prime}\right)\right\rangle_{f_{p}(t)}$. For an infinitely small $f_{p}(t)$, we define the response function $R_{A}\left(t^{\prime}, t\right)$ as

$$
\left\langle A\left(t^{\prime}\right)\right\rangle_{f_{p}(t)}-\left\langle A\left(t^{\prime}\right)\right\rangle=\int_{0}^{t^{\prime}} d t R_{A}\left(t^{\prime}, t\right) f_{p}(t) .
$$

By using (26), we obtain

$$
R_{A}\left(t^{\prime}, t\right)=\left\langle A\left(t^{\prime}\right) i \pi(t)\right\rangle
$$

for $t^{\prime} \geq t$. From this expression, one may call $\pi$ the "response variable". Furthermore, from (23), we also derive

$$
\left\langle A\left(t^{\prime}\right) i \pi(t)\right\rangle=\frac{1}{2 L}\left\langle A\left(t^{\prime}\right)\left(\frac{d V}{d t}-L \frac{d S\left(E_{\mathrm{tot}}-p_{\mathrm{ex}} V, V\right)}{d V}\right)\right\rangle .
$$

\section{Symmetry}

To explore a symmetry property, we map the MSRJD formalism to Hamilton mechanics by setting $p=i \pi$ and $q=V$ in (25). We have

$$
I_{\mathrm{MSR}}=-\int_{0}^{t_{f}} d t\left[p \frac{d q}{d t}-H(p, q)\right]
$$

with

$$
H(p, q)=L p^{2}+L p \frac{d S}{d q}-\frac{1}{2} \frac{d^{2} S}{d q^{2}} .
$$

For a more detailed discussion on mapping, see [10, 33]. Next, we consider the Hamiltonian system with $H(q, p)$. From the Poisson bracket

$$
\begin{aligned}
& \{q, p\}_{\mathrm{PB}}=1, \\
& \{q, q\}_{\mathrm{PB}}=\{p, p\}_{\mathrm{PB}}=0,
\end{aligned}
$$


we obtain

$$
\begin{aligned}
& \{S, p\}_{\mathrm{PB}}=\frac{d S}{d q}, \\
& \{S, q\}_{\mathrm{PB}}=0 .
\end{aligned}
$$

Therefore, $S$ is interpreted as the generator of the following transformation:

$$
\begin{aligned}
& q \rightarrow q, \\
& p \rightarrow p+\eta \frac{d S}{d q},
\end{aligned}
$$

where $\eta$ is a dimensionless infinitesimal transformation parameter. Note here that the transformation is defined for the system irrespective of symmetry.

Here, we consider the variation of the MSRJD action (25) for the transformation (36) and (37), which is calculated as

$$
\begin{aligned}
\delta I_{\mathrm{MSR}} & =-\eta \int_{0}^{t_{f}} d t\left[\frac{d q}{d t} \frac{d S}{d q}-L\left(\frac{d S}{d q}\right)^{2}-2 L p \frac{d S}{d q}\right] \\
& =-\eta \int_{0}^{t_{f}} d t\left[\frac{d S}{d t}-L\left(\frac{d S}{d q}\right)^{2}-2 L p \frac{d S}{d q}\right]
\end{aligned}
$$

where we have used

$$
\frac{d S}{d t}=\frac{d q}{d t} \frac{d S}{d q}
$$

We focus on restricted trajectories near equilibrium, i.e., the trajectories that satisfy

$$
\begin{aligned}
& \frac{d S}{d q}=O(\epsilon), \\
& p=O(\epsilon) .
\end{aligned}
$$

For such restricted trajectories near the equilibrium, we find

$$
\delta I_{\mathrm{MSR}}=-\eta \int_{0}^{t_{f}} d t\left[\frac{d S}{d t}+O\left(\epsilon^{2}\right)\right]
$$

Because $\delta I_{\mathrm{MSR}}$ is written as the time integration of the total derivative of a function, we identify the symmetry for the transformation given by (36) and (37). Based on Noether's theorem that we review below, we can claim that $S$ is the Noether invariant for the system we study.

Now, we derive Noether's theorem for the action of the form

$$
I_{\mathrm{MSR}}[p, q]=-\int_{0}^{t_{f}} d t\left[p \frac{d q}{d t}-H(p, q)\right]
$$

For a general transformation

$$
\begin{aligned}
& p \rightarrow p+\eta \delta p, \\
& q \rightarrow q+\eta \delta q,
\end{aligned}
$$

the variation of the action is calculated as

$$
\delta I_{\mathrm{MSR}}=-\eta \int_{0}^{t_{f}} d t\left[\frac{d}{d t}(p \delta q)+\delta p\left(\frac{d q}{d t}-\frac{\partial H}{\partial p}\right)-\delta q\left(\frac{d p}{d t}+\frac{\partial H}{\partial q}\right)\right] .
$$

Such an action is called symmetric when the variation $\delta I$ can be written as the time integral of the total time derivative of some quantity $\Psi$ :

$$
\delta I=-\eta \int_{0}^{t_{f}} d t \frac{d \Psi}{d t}
$$


Then, we have the relation

$$
\int_{0}^{t_{f}} d t\left[\frac{d}{d t}(p \delta q)+\delta p\left(\frac{d q}{d t}-\frac{\partial H}{\partial p}\right)-\delta q\left(\frac{d p}{d t}+\frac{\partial H}{\partial q}\right)\right]=\int_{0}^{t_{f}} d t \frac{d \Psi}{d t}
$$

Because the second and the third terms vanish along solution trajectories of the Hamilton equation, we obtain that

$$
\int_{0}^{t_{f}} d t\left[\frac{d}{d t}(\Psi-p \delta q)\right]=0
$$

holds along the solution trajectories. Therefore, the quantity $Q \equiv \Psi-p \delta q$ is the Noether invariant.

For the transformation given by (36) and (37), the above quantities should be read as

$$
\begin{aligned}
\delta q & =0, \\
\delta p & =\frac{d S}{d V}, \\
\Psi & =S .
\end{aligned}
$$

Therefore, the Noether invariant is the thermodynamic entropy:

$$
Q=S \text {. }
$$

At the end of this section, we present another form of the action $I_{S}$ in terms of the entropy $S$. Using the transformation from $V$ to $S$, we obtain

$$
I_{S}=\int_{0}^{t_{f}} d t\left[-i \theta\left(\frac{d S}{d t}-L\left(\frac{d S}{d V}\right)^{2}\right)-L\left(\frac{d S}{d V}\right)^{2} \theta^{2}-\frac{1}{2} \frac{d^{2} S}{d V^{2}}\right],
$$

where $\theta=(d V / d S) \pi$. In this representation, the transformation is given as the uniform translation of $\theta$ :

$$
\begin{aligned}
& \theta \rightarrow \theta+i \eta, \\
& S \rightarrow S .
\end{aligned}
$$

Therefore, for trajectories satisfying $d S / d V=O(\epsilon)$, the action (55) possesses the symmetry for this transformation.

\section{Identity}

We set $\mathcal{O}=1$ in (26) and change the integral variable $\pi$ as

$$
\pi^{\prime}(t)=\pi(t)+i \eta \frac{d S}{d V}(t)
$$

Then, we have

$$
\begin{aligned}
1 & =\int \mathcal{D} \pi^{\prime} \mathcal{D} V e^{I_{\mathrm{MSR}}\left[V, \pi^{\prime}\right]}, \\
& =\int \mathcal{D} \pi \mathcal{D} V e^{I_{\mathrm{MSR}}[V, \pi]+\delta I_{\mathrm{MSR}},} \\
& =1+\left\langle\delta I_{\mathrm{MSR}}\right\rangle .
\end{aligned}
$$

We thus obtain

$$
\left\langle\delta I_{\mathrm{MSR}}\right\rangle=0,
$$


where $\delta I_{\mathrm{MSR}}$ is given by (39).

Substituting (39) in (61), we obtain

$$
\left\langle S\left(t_{f}\right)-S(0)\right\rangle=L \int_{0}^{t_{f}} d t\left\langle\left(\frac{d S}{d V}\right)^{2}\right\rangle+2 L \int_{0}^{t_{f}} d t\left\langle i \pi \frac{d S}{d V}\right\rangle,
$$

which corresponds to the Ward-Takahashi identity in quantum field theory. Because the right-hand side comes from the symmetry breaking terms, (62) indicates that the increasing entropy arises from the symmetry breaking. It should also be noted that (62) can be derived by setting $A=d S / d V$ in (29).

Because in the quasi-static limit $\epsilon \rightarrow 0,\left\langle S\left(t_{f}\right)-S(0)\right\rangle=O(\epsilon)$ holds from Noether's theorem, we obtain

$$
\left\langle\left(\frac{d S}{d V}\right)^{2}\right\rangle+2\left\langle i \pi \frac{d S}{d V}\right\rangle=O\left(\epsilon^{2}\right)
$$

Next, we write the equation for $S$ as

$$
\frac{d S}{d t}=L\left(\frac{d S}{d V}\right)^{2}+L \frac{d^{2} S}{d V^{2}}+\frac{d S}{d V} \cdot \sqrt{2 L} \xi
$$

where $\cdot$ is the ito product. Taking the expectation, we have

$$
\left\langle\frac{d S}{d t}\right\rangle=L\left\langle\left(\frac{d S}{d V}\right)^{2}\right\rangle+L\left\langle\frac{d^{2} S}{d V^{2}}\right\rangle
$$

By integrating this relation over the time interval $\left[0, t_{f}\right]$ and comparing the result with (62), we obtain

$$
\left\langle i \pi(t) \frac{d S}{d V}\right\rangle=\frac{1}{2}\left\langle\frac{d^{2} S}{d V^{2}}\right\rangle
$$

By combining (63) with (66), we have

$$
\left\langle\left(\frac{d S}{d V}\right)^{2}\right\rangle+\left\langle\frac{d^{2} S}{d V^{2}}\right\rangle=O\left(\epsilon^{2}\right)
$$

which corresponds to the fluctuation-response relation at equal time. To conclude, the symmetry breaking terms lead to the fluctuation-response relation in the quasi-static limit $\epsilon \rightarrow 0$.

For general non-quasi-static processes, from the second law of thermodynamics (17), we find that

$$
\left\langle S\left(t_{f}\right)-S(0)\right\rangle=L \int_{0}^{t_{f}} d t\left\langle\left(\frac{d S}{d V}\right)^{2}\right\rangle+L \int_{0}^{t_{f}} d t\left\langle\frac{d^{2} S}{d V^{2}}\right\rangle>0 .
$$

This means that the violation of the fluctuation-response relation is related to the entropy production, which corresponds to the Harada-Sasa relation for Langevin equations describing systems in contact with a heat bath 34, 35. Furthermore, from the convexity of the entropy function, we find that $d^{2} S / d V^{2}<0$. By substituting this relation, we obtain

$$
\left\langle S\left(t_{f}\right)-S(0)\right\rangle<L \int_{0}^{t_{f}} d t\left\langle\left(\frac{d S}{d V}\right)^{2}\right\rangle,
$$

which represents an upper bound for the entropy production. We also have a lower bound

$$
L \int_{0}^{t_{f}} d t\left\langle\frac{d^{2} S}{d V^{2}}\right\rangle\left\langle\left\langle S\left(t_{f}\right)-S(0)\right\rangle .\right.
$$

These inequalities may have some relevance to the trade-off relations, including the thermodynamic uncertainty relation [36, 37. 
Thermodynamic entropy as a Noether invariant in a Langevin equation

\section{Concluding remarks}

Although we have studied a rather simple example, our arguments are quite general. First, we write a Langevin equation for a complete set of slow variables in a thermally isolated system following the Onsager theory. Then, we can analyze the Langevin equation, similar to the specific model we studied, and obtain the basically same result for the symmetry property.

However, one may be more familiar with Langevin equations describing stochastic processes in systems with a heat bath. A typical and standard example of such a system is a Brownian particle under an external control. For this case, the entropy production becomes zero in quasi-static processes. Thus, an interesting future problem is to formulate such property as the result of Noether's theorem.

Next, we compare our result with those obtained in the previous studies [11, 12. For isolated quantum many body systems with an external step protocol parameter, the effective action possesses a symmetry for a uniform translation of the canonical conjugate variable of the entropy [12. The uniform transformation is rewritten as (10) for a saddle point solution of the effective action

$$
I_{Q M}=\int_{0}^{t_{f}} d t\left[-\frac{i}{\hbar} E-i \theta \dot{S}\right],
$$

where $E, S$ and $\theta$ are the energy, the entropy, and the canonical conjugate variable to $S$, respectively. Although the transformation is the same as that in our result, our transformation cannot be rewritten as the non-uniform time translation (11) that is found in 11]. The difference comes from the fact that the energy term is absent in (55).

As a final remark, we notice that the action of the Langevin equation is a different concept from the one of the quantum dynamics. The action of the Langevin equation comes from a transition probability, while the action of the quantum dynamics comes from the time evolution of a state vector. The action for the classical mechanics is naturally understood from the quantum mechanics. Therefore, we expect that there exists another type of action which describes a quantum path integral in the thermodynamic state space. Motivated by actions (55) and (71), we conjecture that an action of such a thermodynamic path integral formulation given in [12 for our system can be written as

$$
I\left[S, \dot{S} ; p_{\mathrm{ex}}\right]=\frac{1}{2} I_{S}\left[S, \dot{S} ; p_{\mathrm{ex}}\right]-\frac{i}{\hbar} \int_{0}^{t_{f}} d t E\left(S ; p_{\mathrm{ex}}\right),
$$

which has the same symmetry as $I_{S}$ for the transformation (56) and (57). This leads to the conclusion that $S$ is the Noether invariant. In addition, from the saddle point $\delta I / \delta S=0$, we have

$$
\frac{d \theta}{d t}=\frac{2}{\hbar \beta}+O(\epsilon),
$$

where we used $d S / d V=O(\epsilon)$ in quasi-static processes. By using (73), the transformation (56) could be rewritten as the non-uniform time translation

$$
t \rightarrow t+2 \eta \hbar \beta+O(\epsilon) \text {. }
$$

We leave the derivation of the action (72) from the thermodynamic path integral formulation of [12] for the future work. 
Thermodynamic entropy as a Noether invariant in a Langevin equation

\section{Acknowledgments}

We thank Y. Hidaka, M. Itami and H. Nakano for useful discussions. The present study was supported by KAKENHI (Nos. 17H01148, 19H05496, 19H05795), by the Zhejiang Provincial Natural Science Foundation Key Project (Grant No. LZ19A050001), and by NSF of China (Grant No. 11674283).

\section{References}

[1] Noether E 1918 Invariante variationsprobleme Nachr. Ges. Wiss. Gttingen 235-257

[2] Nambu Y and Jona-Lasinio G 1961 Dynamical model of elementary particles based on an analogy with superconductivity. I. Phys. Rev., 122(1) 345

[3] Goldstone J 1961 Field theories with superconductor solutions Il Nuovo Cimento 19(1) 154-164

[4] Goldstone J, Salam A, and Weinberg S 1962 Broken symmetries Phys. Rev. 127(3) 965

[5] Ward J C 1950 An identity in quantum electrodynamics Phys. Rev. 78 182-182

[6] Takahashi Y 1957 On the generalized ward identity Il Nuovo Cimento (1955-1965) 6(2) 371-375

[7] Hidaka Y 2013 Counting rule for Nambu-Goldstone modes in nonrelativistic systems Phys. Rev. Lett. 110091601

[8] Watanabe H and Murayama H 2012 Unified description of Nambu-Goldstone bosons without Lorentz invariance. Phys. Rev. Lett. 108251602

[9] Hayata T and Hidaka Y 2015 Dispersion relations of Nambu-Goldstone modes at finite temperature and density Phys. Rev. D 91056006

[10] Minami Y and Hidaka Y 2018 Spontaneous symmetry breaking and Nambu-Goldstone modes in dissipative systems Phys. Rev. E 97(1) 012130

[11] Sasa S and Yokokura Y 2016 Thermodynamic entropy as a Noether invariant Phys. Rev. Lett. 116(14) 140601

[12] Sasa S, Sugiura S, Yokokura Y 2019 Thermodynamical path integral and emergent symmetry Phys. Rev. E 99(2) 022109

[13] Zwanzig R 1961 Memory effects in irreversible thermodynamics, Phys. Rev. 124 983-992

[14] Onsager L 1931 Reciprocal relations in irreversible processes. I. Phys. Rev. 37 405-426

[15] Onsager L 1931 Reciprocal relations in irreversible processes. II. Phys. Rev. 38 2265-2279

[16] Casimir H B G 1945 On Onsager's principle of microscopic reversibility Rev. Mod. Phys. 17 $343-350$

[17] Kubo R 1966 The fluctuation-dissipation theorem Rep. Prog. Phys. 29(1) 255

[18] Gozzi E 1984 Onsager principle of microscopic reversibility and supersymmetry Phys. Rev. D $301218-1227$

[19] Gao P and Liu H 2018 Emergent supersymmetry in local equilibrium systems JHEP 2018(1) 40

[20] Anosov D V 1960 Averaging in systems of ordinary differential equations with rapidly oscillating solutions Izv. Akad. Nauk SSSR ser. mat. 24721

[21] Kasuga T 1961 On the adiabatic theorem for the Hamiltonian system of differential equations in the classical mechanics. I Proceedings of the Japan Academy 37, 366

[22] Ott E 1979 Goodness of ergodic adiabatic invariants, Phys. Rev. Lett. 421628 (1979).

[23] Jarzynski C 1993 Multipule-time-scale approach to ergodic adiabatic systems: Another look, Phys. Rev. Lett. 71, 839

[24] Jarzynski C 1997 Nonequilibrium equality for free energy differences Phys. Rev. Lett. 78 26902693

[25] Martin P C, Siggia E D, and Rose H A 1973 Statistical dynamics of classical systems Phys. Rev. A 8 423-437

[26] Janssen H K 1976 On a Lagrangean for classical field dynamics and renormalization group calculations of dynamical critical properties Zeitschrift für Physik B Condensed Matter 23(4) $377-380$

[27] Dominicis C D 1976 Technics of field renormalization and dynamics of critical phenomena $J$. Phys.(Paris), Colloq C1-247

[28] Dominicis C D 1978 Dynamics as a substitute for replicas in systems with quenched random impurities Phys. Rev. B 18 4913-4919

[29] Weinberg S 1996 The Quantum Theory of Fields, Vol. II Cambridge University Press

[30] Aron C, Barci D G, Cugliandolo L F, Arenas Z G, and Lozano G S 2016 Dynamical symmetries of Markov processes with multiplicative white noise J. Stat. Mech.: Theory Exp. 2016(5) 053207 
[31] Aron C, Biroli G, and Cugliandolo L F 2018 (non) equilibrium dynamics: a (broken) symmetry of the Keldysh generating functional SciPost Physics 4(1) 008

[32] Seifert U 2012 Stochastic thermodynamics, fluctuation theorems and molecular machines Rep. Prog. Phys. 75(12) 126001

[33] Justin J Z 1989 Quantum field theory and critical phenomena Clarendon Oxford

[34] Harada T and Sasa S 2005 Equality connecting energy dissipation with a violation of the fluctuation-response relation Phys. Rev. Lett. 95130602

[35] Harada T and Sasa S 2006 Energy dissipation and violation of the fluctuation-response relation in nonequilibrium Langevin systems Phys. Rev. E 73026131

[36] Barato A C and Seifert U 2015 Thermodynamic uncertainty relation for biomolecular processes Phys. Rev. Lett. 114(15) 158101

[37] Gingrich T R, Horowitz J M, Perunov N, and England J L 2016 Dissipation bounds all steadystate current fluctuations Phys. Rev. Lett. 116(12) 120601 